\title{
Reflected Near-field Blast Pressure Measurements Using High Speed Video
}

\author{
S. E. Rigby ${ }^{1}$ (D) R. Knighton ${ }^{1} \cdot$ S. D. Clarke ${ }^{1} \cdot$ A. Tyas $^{1,2}$
}

Received: 2 September 2019 / Accepted: 1 June 2020 / Published online: 1 July 2020

(C) The Author(s) 2020

\begin{abstract}
Background: The design and analysis of protective systems requires a detailed understanding of, and the ability to accurately predict, the distribution of pressure loads acting on an obstacle following an explosive detonation. In particular, there is a pressing need for accurate characterisation of blast loads in the region very close to a detonation, where even small improvised devices can produce serious structural or material damage. Objective: Accurate experimental measurement of these near-field blast events, using intrusive methods, is demanding owing to the high magnitudes ( $>100 \mathrm{MPa}$ ) and short durations $(<1 \mathrm{~ms})$ of loading. The objective of this article is to develop a non-intrusive method for measuring reflected blast pressure distributions using image analysis. Methods: This article presents results from high speed video analysis of nearfield spherical PE4 explosive blasts. The Canny edge detection algorithm is used to track the outer surface of the explosive fireball, with the results used to derive a velocity-radius relationship. Reflected pressure distributions are calculated using this velocity-radius relationship in conjunction with the Rankine-Hugoniot jump conditions. Results: The indirectly measured pressure distributions from high speed video are compared with directly measured pressure distributions and are shown to be in good qualitative agreement with respect to distribution of reflected pressures, and in good quantitative agreement with peak reflected pressures (within 10\% of the maximum recorded value). Conclusions: The results indicate that it is possible to accurately measure blast loads in the order of $100 \mathrm{~s} \mathrm{MPa} \mathrm{using} \mathrm{techniques} \mathrm{which} \mathrm{do} \mathrm{not} \mathrm{require} \mathrm{sensitive} \mathrm{recording} \mathrm{equipment}$ to be located close to the source of the explosion.
\end{abstract}

Keywords Blast $\cdot$ High speed video $\cdot$ Image tracking $\cdot$ Near-field $\cdot$ Reflected pressure distribution

\section{Introduction}

Accurate quantification of blast wave parameters remains a significant challenge to the research community, in particular determination of the spatial distribution of pressure imparted to an obstacle located a short distance from the explosive. Semi-empirical methods for predicting blast pressure loads, e.g. the well-established Kingery \& Bulmash [1] scaled distance relationships, are largely derived from experiments conducted in the mid- $20^{\text {th }}$ century [2]. Whilst these predictive methods have been shown

$\triangle$ S. E. Rigby

sam.rigby@shef.ac.uk

1 Department of Civil \& Structural Engineering, University of Sheffield, Mappin Street, Sheffield, S1 3JD, UK

2 Blastech Ltd., The Innovation Centre, 217 Portobello, Sheffield, S1 4DP, UK to be highly accurate for geometrically simple far-field situations [3-5], blast parameter relationships in the nearfield are derived from very few experimental measurements [6] and are not able to accurately represent the complex situation that occurs in the early stages of an explosion [7, 8]. Consequently, numerical analyses in this region demonstrate considerable deviation from semi-empirical predictions [9].

Direct measurement of near-field blast pressures requires robust, hardened apparatus that can survive explosive loading in the order of $100 \mathrm{~s} \mathrm{MPa}$, yet is sensitive enough to resolve spatial and temporal features that vary in the $\mathrm{mm}$ and $\mu$ s range respectively. Whilst experimental techniques have recently been developed for measuring surface loads resulting from explosive detonations (e.g. [10], and the apparatus developed by Clarke et al. [11] which provides comparative results in this article), these methods are intrusive. There is still a need to develop non-intrusive methods which do not require sensitive recording equipment to be located close to the source of the explosion, as this 
may adversely affect the accuracy and longevity of an experimental approach.

Photo-optical techniques have historically been used to record shock front arrival times and velocities, which enabled properties such as pressure and density to be calculated for a freely expanding blast wave [12, 13] by relating particle velocities to shock pressures using the Rankine-Hugoniot jump conditions. Recent advancements in high speed video (HSV) analysis have enabled researchers to further study the properties of blast waves using imaging techniques. Biss and McNesby [14] used schlieren flow visualization and high-speed digital imaging to optically measure the temporal decay of incident blast waves in the 100s kPa range. Hargather \& Settles [15] and Hargather [16] used a similar technique to establish the relationship between incident shock pressure and distance for a number of explosive types.

High speed video images are obscured by the intense light released after detonation. Hence, the aforementioned research and other optical-based image tracking studies, e.g. [17] and [18], focus on the intermediate to far-field region where the shock front is freely expanding and has detached from the detonation product fireball. Alternative approaches use pressurised glass spheres [19] or detonation transmission tubing [20] in place of the explosive. In the near-field, however, the detonation product fireball is still rapidly expanding and remains attached to the surrounding layer of compressed air [21]. Thus, in the early stages of fireball expansion, measurement of the velocity of the outer surface of the detonation product fireball equates to measurement of the shock velocity, as is assumed in this article. Schlieren methods are generally not suited for nearfield situations as the intensity of light from the fireball is such that the pressure boundary is indistinguishable from the surrounding air [22].

McNesby et al. [23, 24] used a framing camera and streak camera techniques to measure the outer surface of the fireball, and again used the Rankine-Hugoniot jump conditions to determine near-field incident shock pressures. Whilst incident shock pressures accurately describe the properties of a blast wave as it propagates unimpeded through free air, when an incident blast impinges on a notionally rigid reflecting surface its pressure is amplified as a result of conservation of mass, momentum and energy at the interface [25]. The transition from incident to reflected pressure conditions can result in an increase in pressure by up to a factor of 20 for near-field explosions [26]. Reflected blast wave parameters represent the loading an object located in the path of the explosion will be subjected to, and therefore the provision of adequate blast protection systems requires a detailed knowledge of reflected blast pressure conditions and how they are distributed across the face of an obstacle.
To date, there have been no studies which have evaluated near-field reflected blast pressure distributions using non-intrusive image-based techniques. This article presents measurements of reflected blast pressures using high speed video data. A free-air velocity-radius relationship is derived from image tracking of the explosive fireball using numerical edge detection techniques. The velocity relationship is then used to derive reflected pressure predictions on a rigid surface using Rankine-Hugoniot jump conditions. Predictions are compared against directly measured pressure distributions and the method presented herein is shown to be an accurate method for determining reflected blast wave parameters (within $10 \%$ of the maximum directly-measured value) through optical measurement of incident conditions only, in the regions of extremely high pressures and shock velocities close to the source of an explosion. The results are also used to make comments on the emergence and growth of turbulent instabilities at the fireball/air interface.

\section{Experimental Work}

Experiments were performed at the University of Sheffield Blast \& Impact Laboratory in Buxton, Derbyshire, UK, using the Characterisation of Blast Loading apparatus [11]. The apparatus comprises a pair of steel fibre and bar reinforced concrete frames, set approximately $1 \mathrm{~m}$ apart (Fig. 1(a)). A $1400 \mathrm{~mm}$ diameter, $100 \mathrm{~mm}$ thick mild steel target spans between the undersides of each frame, and acts as a nominally rigid target to reflect the blast pressures when an explosive charge is detonated some stand-off distance beneath the centre of the plate.

The plate is drilled through its thickness, with one $10.5 \mathrm{~mm}$ diameter central hole, and four holes ${ }^{1}$ at each radial distance of $25 \mathrm{~mm}, 50 \mathrm{~mm}, 75 \mathrm{~mm}$, and $100 \mathrm{~mm}$ from the plate centre (Fig. 1(b)). These holes form two orthogonal arrays passing through the centre, and enable pressure to be measured at 17 locations within the central $200 \mathrm{~mm}$ diameter region of the target plate. $3.25 \mathrm{~m} \mathrm{long}$, $10.0 \mathrm{~mm}$ diameter EN24T steel Hopkinson pressure bars (HPBs, after [29]) are suspended from their distal ends and inserted through the holes such that their faces lie flush with the face of the target plate.

Kyowa KSP-2-120-E4 semi-conductor strain gauges mounted in pairs on the perimeter of each HPB allow axial strain to be recorded and thus the reflected pressure

\footnotetext{
${ }^{1}$ Previous experimental work into buried explosives [28] has shown the loading distribution is influenced by the highly irregular soil/air interface, and hence highlighted the need for multiple measurements at a given distance from the explosive/target centre. In this study, three repeat tests and four discrete measurements at each non-central radial ordinate provide twelve data points from which an average can be taken.
} 

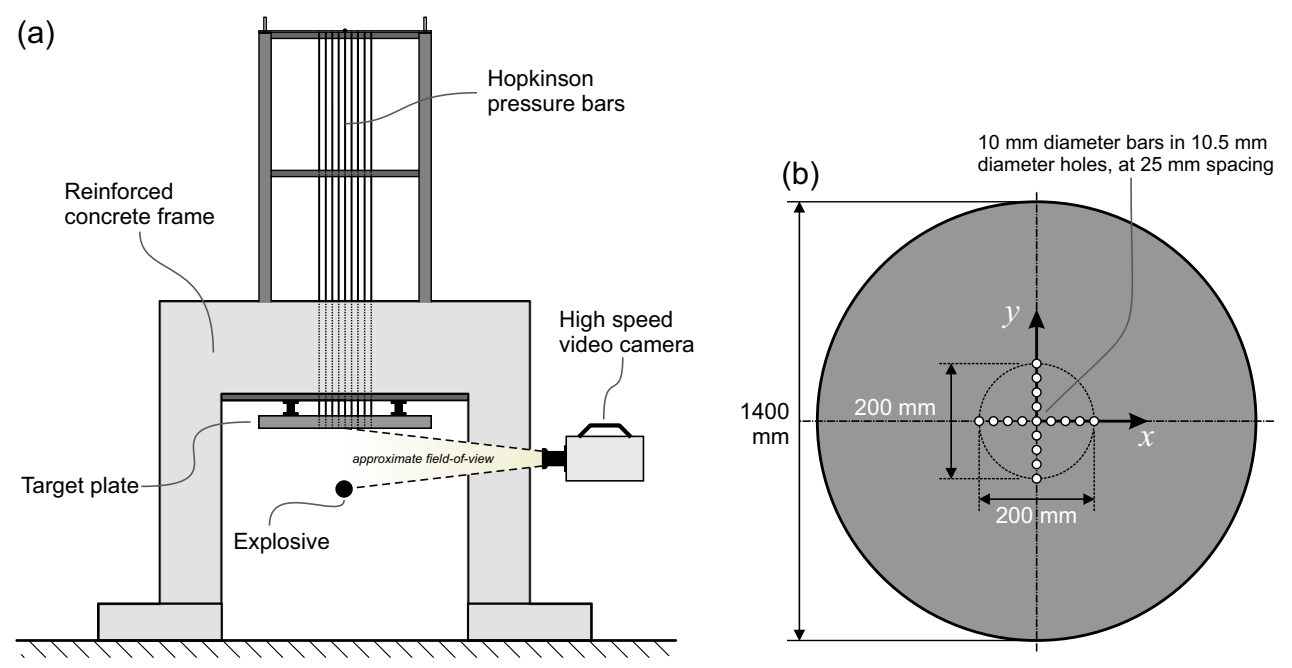

Fig. 1 Schematic of Characterisation of Blast Loading apparatus [not to scale]: a) elevation; b detailed plan view of target plate showing bar arrangement (adapted from [27]). Note, camera positioning is indicative

history at the bar face to be calculated. HPB strain data were recorded using 14-Bit digital oscilloscopes at a sample rate of $3.12 \mathrm{MHz}$, although only peak pressure values are required in this study.

$100 \mathrm{~g}$ PE4 charges ( $24.6 \mathrm{~mm}$ radius) were formed into spheres using bespoke 3D printed charge moulds and were suspended on a glass-fibre weave fabric 'drumskin' (25 $\mathrm{g} / \mathrm{m}^{2}$ area density), held taught in a steel ring and suspended from the test frame on adjustable screws. The charges were aligned with the centre of the plate using an alignment laser, and were centrally detonated using Nitronel MS 25 non-electronic shock-tube detonators ( $0.7 \mathrm{~g}$ PETN) inserted through the bottom face of the charge. PE4 is an RDX-based plastic explosive comprising $87 \%$ RDX and $13 \%$ mineral oil binder [30], and is the UK equivalent of C4 [31].

A Photron FASTCAM SA-Z high speed video camera was situated in a protective housing, located a short distance from the test apparatus. The camera was fitted with a $105 \mathrm{~mm}$ Nikon lens and an infra-red filter was attached to the inner surface of a polycarbonate window through which the tests were filmed. The tests were self-illuminated by the incandescence of the detonation product cloud, and were recorded at $160,000 \mathrm{fps}$ with $280 \times 256$ pixel resolution, $\mathrm{f} / 8$ aperture and $0.25 \mu$ s shutter speed. The camera was positioned approximately level in height with the centre of the charge, with the vertical field-of-view set between the charge centre and the underside of the target plate $(160,000 \mathrm{fps}$ is the maximum frame rate achievable with this field-of-view). Video recording was synchronised with the detonation by triggering the camera off a voltage drop in a breakwire wrapped around the detonator. Post-processing of preliminary video data confirmed that lens distortion was negligible for the current test setup.
Three tests were performed as part of this study, with the explosive placed at a stand-off (distance from the charge centre to the plate) of $380 \mathrm{~mm}$. All tests were conducted on the same day, under the same lighting conditions, and the camera was only moved for small field-of-view adjustments. Additionally, results from the three $100 \mathrm{~g}$ spherical PE4 tests at $80 \mathrm{~mm}$ stand-off in [32] are used for comparative purposes.

\section{High Speed Video Edge Detection}

\section{Edge Detection Algorithms}

Canny [33] devised a numerical algorithm particularly suited for edge detection in images with high levels of background noise [34]. This is available in MatLab as the (pre-existing) edge function. A low-pass Gaussian filter (default standard deviation $\sigma=\sqrt{2}$ ) is first applied to the image to remove noise. Following this, intensity gradients are calculated through convolution of four filters used to detect horizontal, vertical and diagonal edge locations. Finally, a two-stage subroutine is implemented. In the first stage, a non-maxima suppression algorithm is applied, which ensures that the central pixel (of the detected edge) has a gradient magnitude larger than the pixels adjacent to the edge. In the second stage, hysteresis thresholding is used to remove noise and suppress weak edges that are not connected to strong edges: a high threshold first identifies strong edges, and a low threshold removes low gradient value pixels that are not attached to the strong edge. Any 
remaining pixels are assigned the value of 1 and comprise an edge, with the remaining pixel values set to 0 . In this study, the user-controlled high and low threshold values were determined iteratively for each frame to ensure the entire edge was detected.

In addition to the Canny [33] algorithm, a binarisation technique was used for the first two frames post-detonation where parts of the image were obscured by the intense flash of light, and reflections of the flash from neighbouring surfaces corrupted the Canny edge detection. This was achieved by modifying the imbinarize MatLab function to apply a threshold to the image. A purpose-written code then located the first pixel above the threshold (when reading from top to bottom) and assigned this a value of 1 to denote the detected edge (with all other pixels in that column set to zero). Edge detection was terminated immediately before the fireball impinged on the target plate, giving a maximum measurable fireball radius of $380 \mathrm{~mm}$. Typically 20-25 frames were analysed at an inter-frame rate of $6.25 \mu \mathrm{s}$.

Figure 2 shows the raw HSV images and Fig. 3 shows the corresponding frames with edge detection algorithms applied. Note: at $t=0$ the binarisation routine was implemented, whereas for all other frames in this figure the Canny approach was used.

\section{Determining Fireball Radius and Velocity}

The pixel height and width was calculated for each image using the known distance between the centre of the charge and the underside of the target $(380 \mathrm{~mm})$. This was calibrated separately for each test to account for slight changes in charge placement and camera positioning, with the centre of the charge calculated by taking the average centre point of the fireball for the first three frames after detonation. Since the tests were self-illuminated by the brightness of the fireball the charge was not visible prior to detonation, and calibrating off a static image taken by a different camera would have introduced errors induced by placement. The average pixel dimension was $1.836 \mathrm{~mm}$ with an associated error of $\pm 0.035 \mathrm{~mm} /$ pixel, i.e. \pm 4 pixels along the $380 \mathrm{~mm}$ calibration length. At the maximum fireball radius, the maximum error is approximately $\pm 7.5 \mathrm{~mm}$, i.e. $\pm 2.0 \%$, which is comparable to the calibration uncertainty in previous published work [20].

The fireball radius was calculated using an in-house code developed by the authors (illustrated in Fig. 4) by discretising the domain into $1^{\circ}$ increments along virtual 'spokes' emanating from the charge centre. First, the centroids of the two edge pixels nearest the spoke were

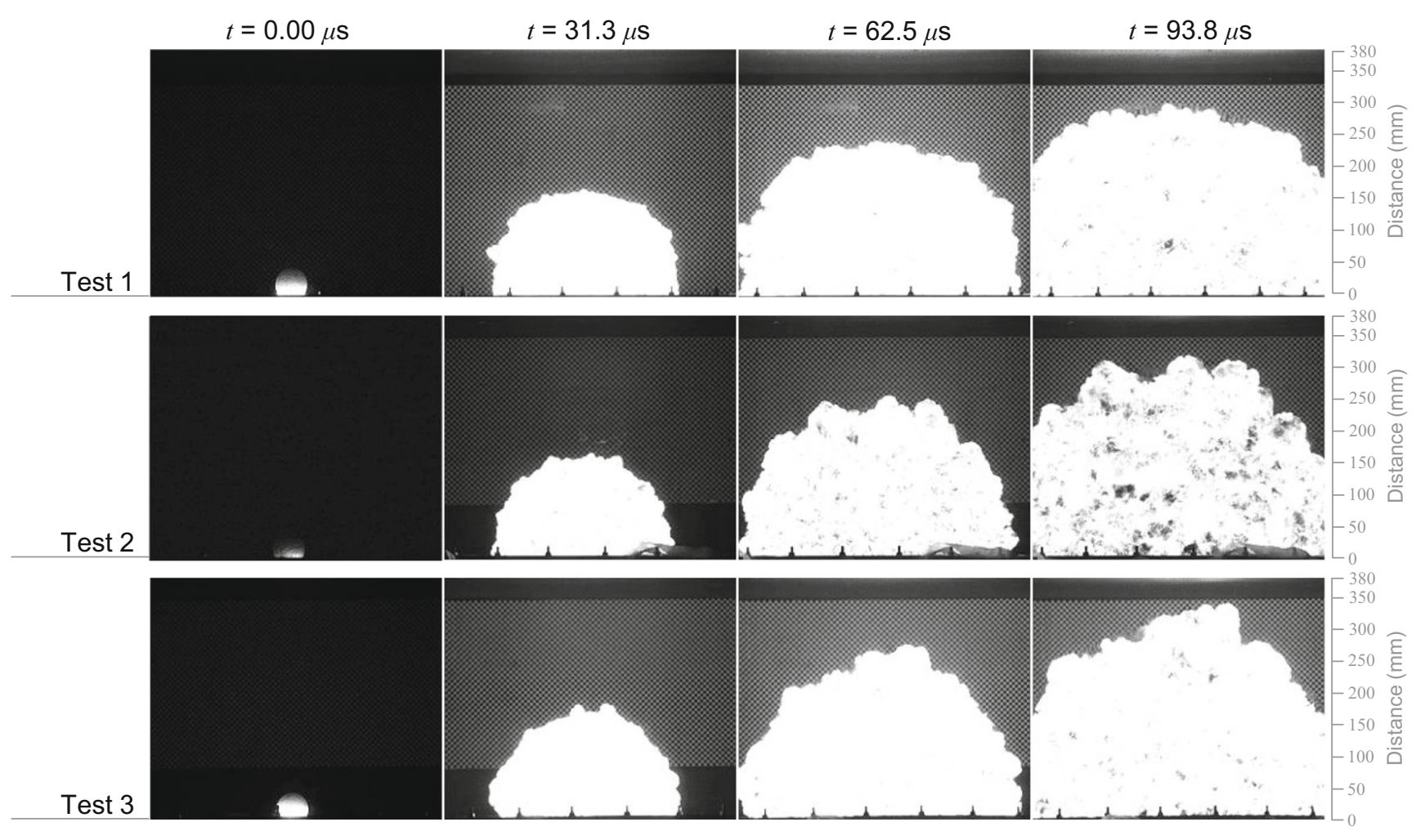

Fig. 2 Raw high speed video stills 


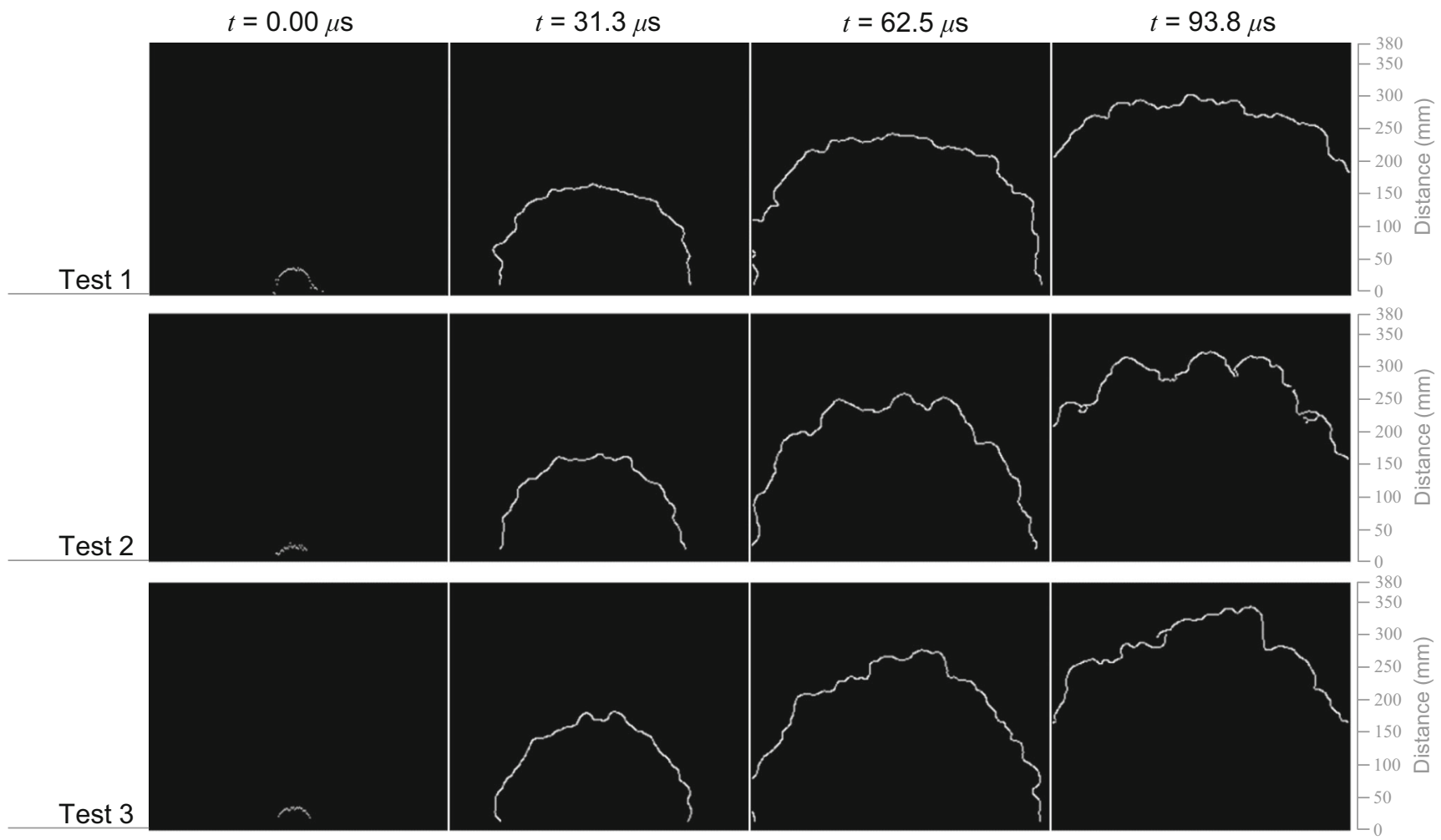

Fig. 3 High speed video stills with edge detection algorithm applied

determined by identifying the two minimum values of $|\phi-\psi|$, where $\phi$ is the angle between the vertical line intersecting the centre of the explosive and the spoke, and $\psi$ is the angle between the vertical and the line connecting the pixel centroid to the charge centre. In Fig. 4, the nearest edge pixels are identified by the angles $\psi_{1}$ and $\psi_{2}$. The position of the fireball edge for that value of $\phi$ is then defined as the point where the spoke and the line connecting the two pixel centroids intersect.
The radial discretisation of $\Delta \phi=1^{\circ}$, for $-90^{\circ} \leq$ $\phi \leq 90^{\circ}$, was judged to provide sufficiently accurate measurements of the outer surface of the fireball given the resolution of the images. A subroutine was written to eliminate any spoke once the fireball surface had reached the edge of the image along that spoke, or if the fireball was judged to have impacted the target between the previous frame and the current one. This provided measurements of the fireball surface for slant distances up to $400 \mathrm{~mm}$
Fig. 4 Method for detecting location of fireball edge along a virtual 'spoke' by identifying neighbouring pixel centroids

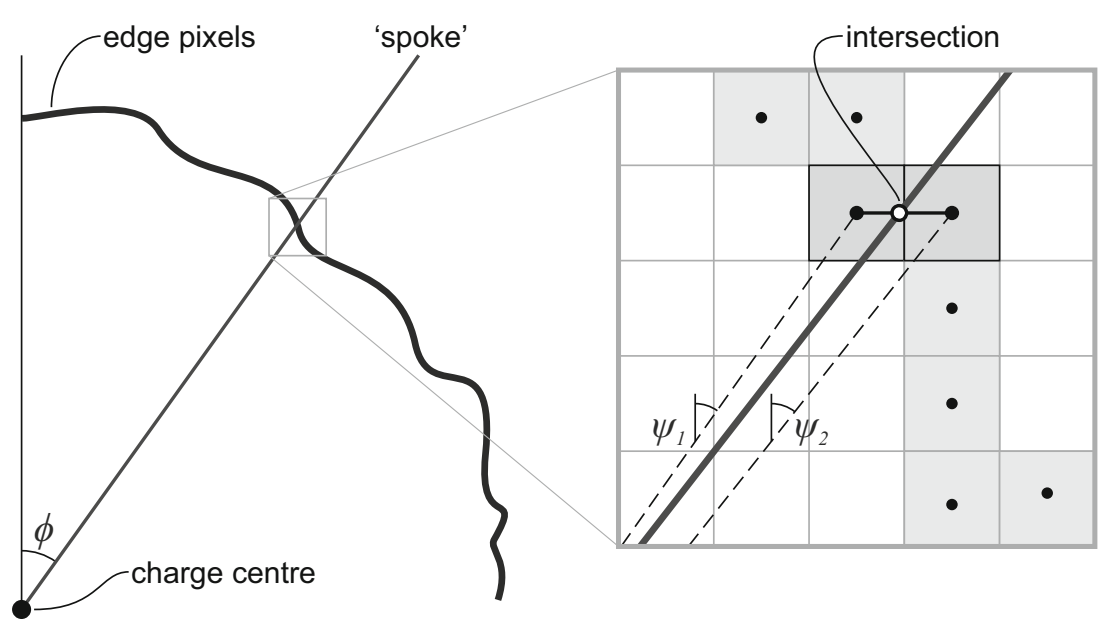


from the charge centre. The velocity along each spoke was determined at the inter-frame midpoint though linear central differencing of the displacement data.

\section{Results and Discussion}

\section{Fireball Radius and Velocity Measurements}

Figures 5 and 6 show fireball surface radius and fireball surface velocity measurements for Tests $1-3$ respectively. The mean displacements and velocities for each individual test are represented by the solid black line, and each spoke is represented by the grey lines in order to provide qualitative information on the degree of spread in the results. Mean fireball surface radius and velocity measurements are compiled in Figs. 5d and 6d.

The fireball radius measurements can be seen to form a tight banding around the mean in the early stages of expansion $(t<30 \mu \mathrm{s})$. After the fireball has expanded approximately $150 \mathrm{~mm}$, i.e. 6 charge radii or a scaled distance of $Z=0.150 /\left(0.1^{1 / 3}\right)=0.32 \mathrm{~m} / \mathrm{kg}^{1 / 3}$, the spread in the measured data begins to increase, albeit gradually, and the data continues to become more widespread until the outer limits of the fireball have propagated $380 \mathrm{~mm}$ and impact the target plate. It should be noted that measurements of decreased velocity are hypothesised to be as a result of light obscuration rather than being a genuine feature of fireball expansion. Given that 180 spokes were analysed in each test, a single spoke being obscured for a single frame is likely to have a minimal effect on the mean velocity.

At the moment of first impact of any part of the fireball on the target plate, the fireball radius typically varies between $300-380 \mathrm{~mm}$, suggesting that there are regions of the fireball/air interface that propagate at considerably higher velocities than the main body of the fireball, which leads to a slight skew in the mean velocity. The velocity data shows sporadic, short duration increases in velocity which then appear to decrease, stabilise, and remain at some value above the mean thereafter. This behaviour is illustrated in Fig. 6b, where the trajectory of a single 'spoke' has been highlighted. It is suggested that this shows evidence of the emergence and growth of Rayleigh-Taylor [35, 36] and Richtmyer-Meshkov [37, 38] surface instabilities (RT and RM respectively). RT instabilities develop when the density gradient and the pressure gradient are in opposite directions, which occurs when the detonation products expand and compresses the surrounding air to the point at which the local air pressure exceeds that in the fireball (which itself remains denser than the air). RM instabilities are generated when a shock passes through inhomogeneous media, e.g. localised inhomogeneities initially caused by RT instabilities [39]. Such instabilities have previously been
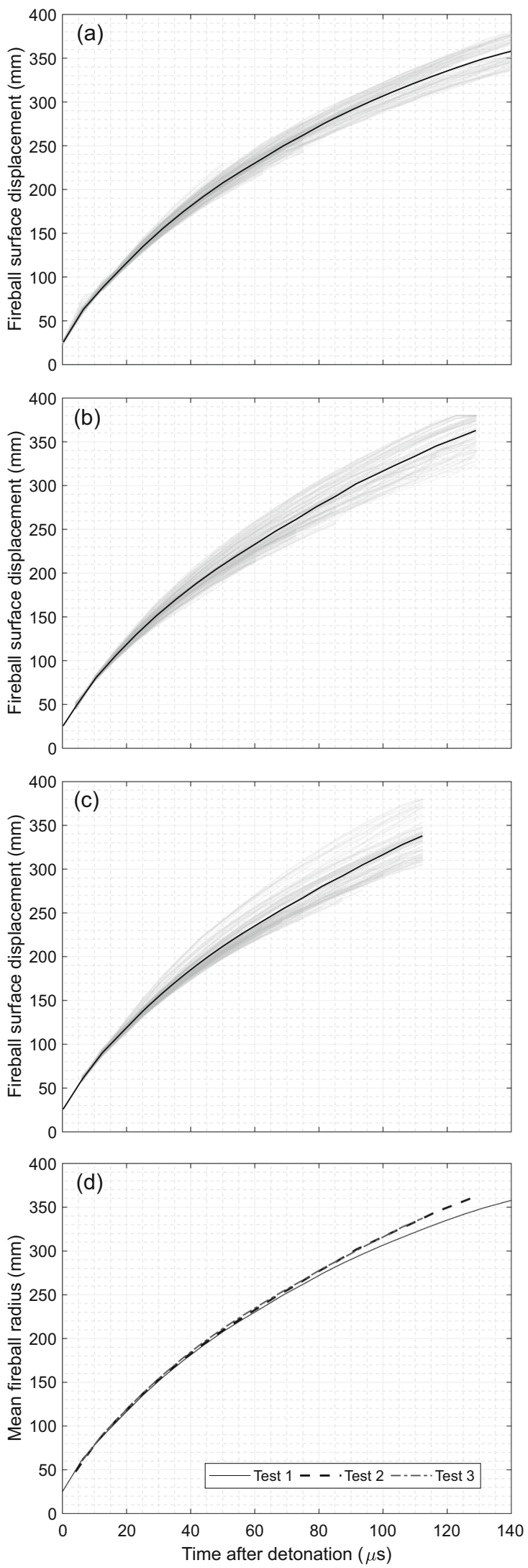

Fig. 5 Fireball radii for Tests 1-3 (a-c) and compiled mean comparison (d) 

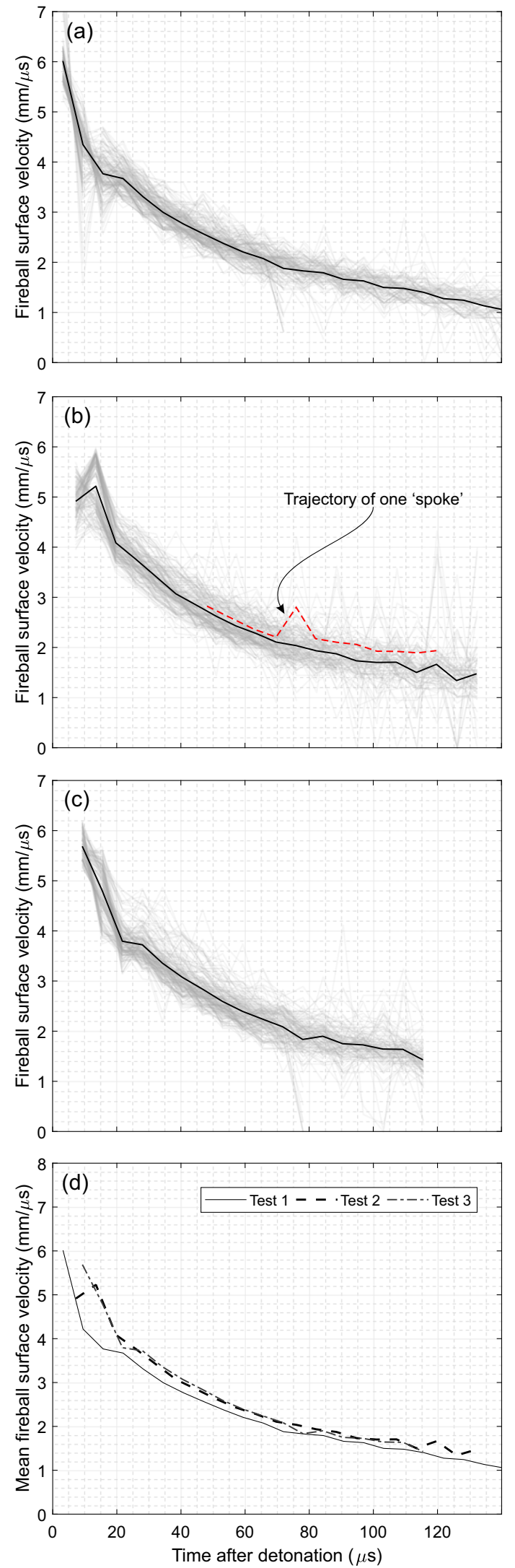

Fig. 6 Fireball surface velocity measurements for Tests 1-3 (a-c) and compiled mean comparison $(\mathbf{d})$

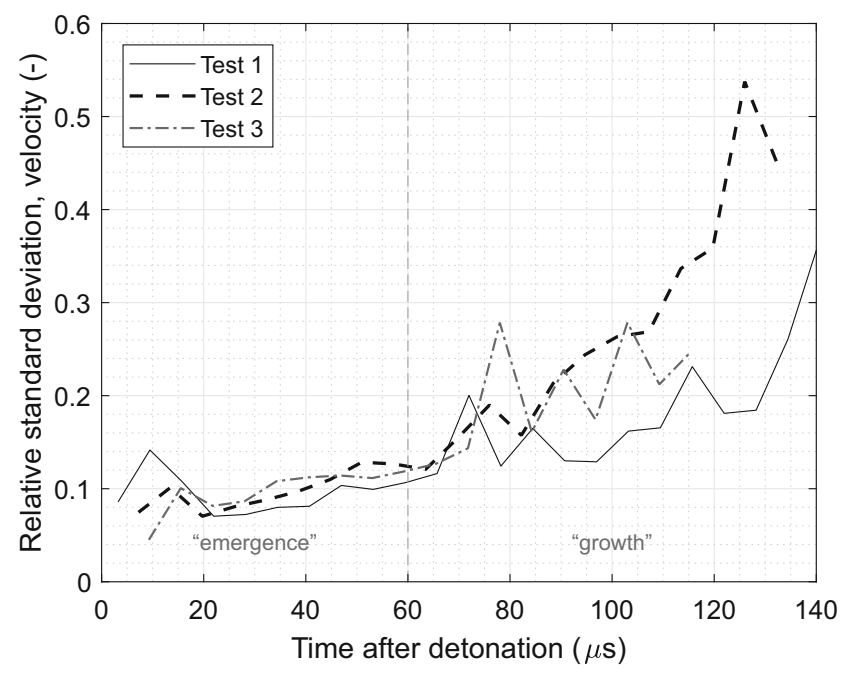

Fig. 7 Variation of velocity with time for all test showing emergence and growth of fireball surface instabilities

observed to significantly influence the localised load on a reflecting surface [40, 41].

These variations in fireball surface velocity are further examined in Fig. 7. Here, the relative standard deviation (RSD $=\sigma / \mu)$ of surface velocity has been calculated at each instant in time using velocity data from all available spokes, and is plotted for each test. It can be seen that the relative standard deviation increases gradually from an initial average value of $8 \%$ at $20 \mu \mathrm{s}$, to an average value of $12 \%$ at $60 \mu \mathrm{s}$ after detonation (corresponding to 3-10 charge radii, with reference to Fig. 5d). Beyond this point the curves begin to rapidly increase and diverge.

It is hypothesised that we are observing two distinct stages of emergent instabilities, which we term: emergence and growth. In the first stage, the emergence of early-time instabilities gives rise to turbulent features which induce low-level and largely deterministic/repeatable variations in fireball surface velocities. This behaviour persists up to a fireball radius of approximately 10 charge radii. Beyond 10 charge radii the second stage is entered, where late-time growth of instabilities gives rise to turbulent features which influence fireball surface velocity in a less deterministic manner. Hence, there is an increased level of inherent stochasticity (i.e. increased and less predictable relative standard deviation) during the growth phase. 10 charge radii corresponds to a scaled distance of $Z=0.246 /\left(0.1^{1 / 3}\right)=$ $0.53 \mathrm{~m} / \mathrm{kg}^{1 / 3}$. This closely matches the value of $Z=$ $0.50 \mathrm{~m} / \mathrm{kg}^{1 / 3}$ proposed by Tyas [30] as the transition from 'extreme' to 'late' near-field regions, based on a collation of data on directly measured peak reflected pressure and peak specific impulse in experiments conducted over a range of scaled distances. Here, loading is described as "relatively consistent" in the extreme near-field, with "large variations in loading" in the late near-field, which appears to be 
similar to the observations of increased variability of fireball surface velocities (for $Z>0.53 \mathrm{~m} / \mathrm{kg}^{1 / 3}$ ) in this article.

\section{Reflected Pressure Calculations from Fireball Velocity}

The radius--time and velocity--time plots in $5 \mathrm{~d}$ and $6 \mathrm{~d}$ were combined to generate the velocity-radius plot shown in Fig. 8, assuming that any error as a result of averaging the data over inter-frame times is minimal. A third-order polynomial equation was fit to the relationship (coefficient of determination, $R^{2}=0.998$ ), as this curve type has previously been shown to most accurately represent experimentally recorded near-field blast wave radius-time data [42]. ${ }^{2}$ Note that this fit type was selected based on its ability to best represent the data rather than being physicsbased. Also shown on the secondary $y$-axis of Fig. 8 is the relative error of the curve fit. Between a fireball radius of 50 and $150 \mathrm{~mm}$ the maximum relative error is $5.2 \%$, whereas from 150 to $400 \mathrm{~mm}$ radius the relative error is within $0.8 \%$.

The polynomial curve fit presented in Fig. 8 provides a relationship to calculate the mean fireball surface velocity, $v(\mathrm{~mm} / \mu \mathrm{s})$, as a function of fireball radius, $r(\mathrm{~mm})$, for $25<r<400 \mathrm{~mm}$ :

$v=-39.54 \mathrm{E}-9 r^{3}+60.47 \mathrm{E}-6 r^{2}-32.22 \mathrm{E}-3 r+7.079$

Dividing through by ambient sonic velocity $(0.34 \mathrm{~mm} / \mu s)$ gives an expression for the incident Mach number, $M_{i}$, as a function of fireball radius:

$M_{i}=-116.3 \mathrm{E}-9 r^{3}+177.8 \mathrm{E}-6 r^{2}-94.76 \mathrm{E}-3 r+20.82$

Consider reflection of a blast wave after it strikes a rigid target, Fig. 9a. The angle of incidence, $\theta$, at a point on the surface (denoted by the letter $A$ in this figure) is defined as the angle between the outward normal of the surface and the direct vector from the explosive charge to that point [43]. Accordingly, $\tan (\theta)=x / y$, where $x$ is the distance along the target to the point of impingement, and $y$ is the distance from the centre of the explosive to the point of impingement (termed stand-off distance).

\footnotetext{
${ }^{2}$ In their study of particle-blast interaction during explosive dispersal of particles and liquids, [42] compared a number of different types of curves and assessed their ability to model the measured shock front trajectories. Namely, they trialled a second order polynomial, a third order polynomial, a 'rational model' (a ratio of linear function and second order polynomial) and the logarithmic decay presented by Dewey [12]. It was found that the third order polynomial offered the most accurate fit, particularly in the near-field, and the authors opted for this fit type for all subsequent analyses in their study. Based on these findings, and particularly given the fact that the current approach focusses on near-field data, a third order polynomial has been adopted in this paper
}

Kinney and Graham [44] outline an analytical procedure based on the Rankine-Hugoniot jump conditions to determine the oblique reflected pressure, $p_{r, \theta}$, acting on a rigid surface following impingement of an incident shock propagating at Mach number $M_{i}$. In the limiting case of normal reflection, i.e. $\theta=0^{\circ}$, the reflected Mach number is given as

$M_{r, \theta=0}=\sqrt{\frac{7 M_{i}^{2}-1}{M_{i}^{2}+5}}$

and the normally reflected overpressure (where the term 'overpressure' refers to the increase in pressure above atmospheric conditions):

$p_{r, \theta=0}=p_{a}\left[\frac{\left(4 M_{i}^{2}-1\right)\left(7 M_{i}^{2}-1\right)}{3\left(M_{i}^{2}+5\right)}-1\right]$,

where $p_{a}$ is ambient pressure. In the limiting case of $\theta=$ $90^{\circ}$, the blast propagates parallel to the surface and incident conditions are maintained: $M_{r, \theta=90}=M_{i}$ and $p_{r, \theta=90}=p_{i}$, where $p_{i}$ is overpressure of the incident wave and is a function of incident Mach number only,

$p_{i}=p_{a} \frac{7\left(M_{i}{ }^{2}-1\right)}{6}$.

Considering impingement of an incident shock non-normal to a rigid surface $\left(0^{\circ}<\theta<90^{\circ}\right)$, as a steady-flow counterpart (see Fig. 9b), oblique reflection generates an intermediate flow stream, such that

$\frac{\tan (\theta-\alpha)}{\tan \theta}=\frac{5+M_{i}{ }^{2}}{6 M_{i}{ }^{2}}$

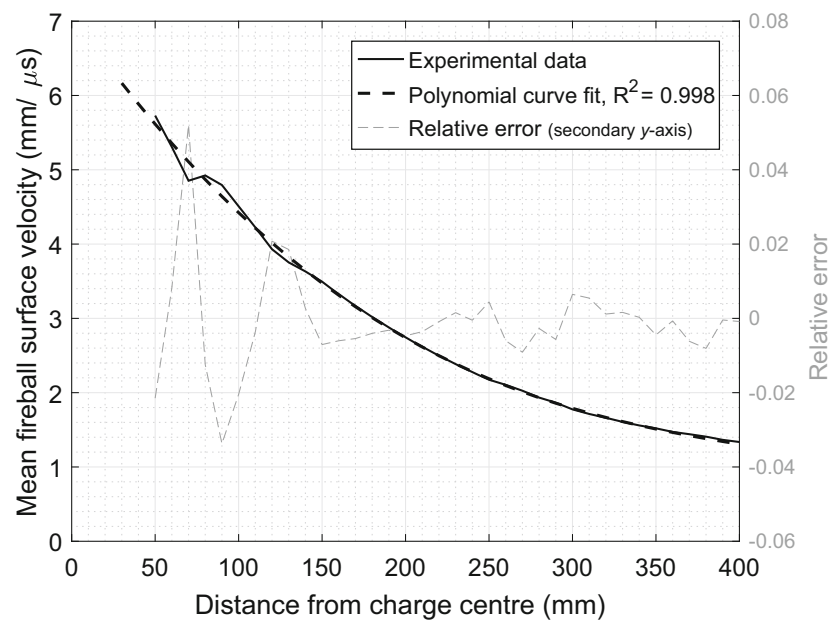

Fig. 8 Velocity-radius relationship and third order polynomial fit to data (equation (2)), with relative error plotted on secondary $y$-axis 
where $\alpha$ is the stream deflection angle, and $M_{2}$ is the stream Mach number, given by

$M_{2} \sin (\theta-\alpha)=\sqrt{\frac{M_{i}^{2}+5}{7 M_{i}^{2}-1}}$

This intermediate stream instantaneously enters the reflected region at an angle $\beta$. Noting that the corresponding stream deflection angle is $\alpha$, the angle of entry, $\beta$, can be determined through iteration of the following relation

$\frac{\tan (\beta-\alpha)}{\tan \beta}=\frac{5+\left(M_{2} \sin \beta\right)^{2}}{6\left(M_{2} \sin \beta\right)^{2}}$

The oblique reflected Mach number, $M_{r, \theta}$, is thus given as

$M_{r, \theta}=M_{2} \sin \beta$

which can be used to determine the oblique reflected overpressure:

$p_{r, \theta}=p_{a}\left[\frac{\left(7 M_{r, \theta^{2}}-1\right)\left(7 M_{i}^{2}-1\right)}{36}-1\right]$

Equation (2) can be used in conjunction with equations (610) to provide pressure predictions along a rigid reflecting surface at any distance $(25<r<400 \mathrm{~mm})$ from the centre of a $100 \mathrm{~g}$ spherical PE4 explosive.

The relationship for oblique reflected Mach number is a non-continuous function of angle of incidence, and is shown in Fig. 9c. A discontinuity occurs at angles of incidence between $40-48^{\circ}$, with the exact value a function of incident Mach number. This point marks the transition from regular to Mach reflection (or 'irregular reflection'), as approximately indicated in Fig. 9a. Mach reflection occurs when the higher pressure (and hence higher velocity) reflected wave coalesces with the incoming incident wave and forms the Mach Stem. The Mach Stem connects the reflecting surface with the point where the incident and reflected waves meet, known as the triple point. Note: the analytical work of [44] described above holds for both regular and Mach reflection.

The previous expressions assume ideal gas behaviour, that is the ratio of specific heats, $\gamma$, remains constant at a value of 1.4. Real gas behaviour begins to deviate from ideal gas behaviour at incident pressures greater than $2 \mathrm{MPa}$ (300 psi), where dissociation and ionisation can lead to a decrease in $\gamma$ [39], however this effect is expected to be minimal in the current study where incident pressures are not expected to exceed $25 \mathrm{MPa}(\sim 200 \mathrm{MPa}$ reflected pressure, assuming a reflection coefficient of 8.0 after [44], where the reflection coefficient is defined as the ratio of the peak magnitudes of reflected and incident pressures). Thus, the simplified constant $-\gamma$ relations are used in this article. (a)
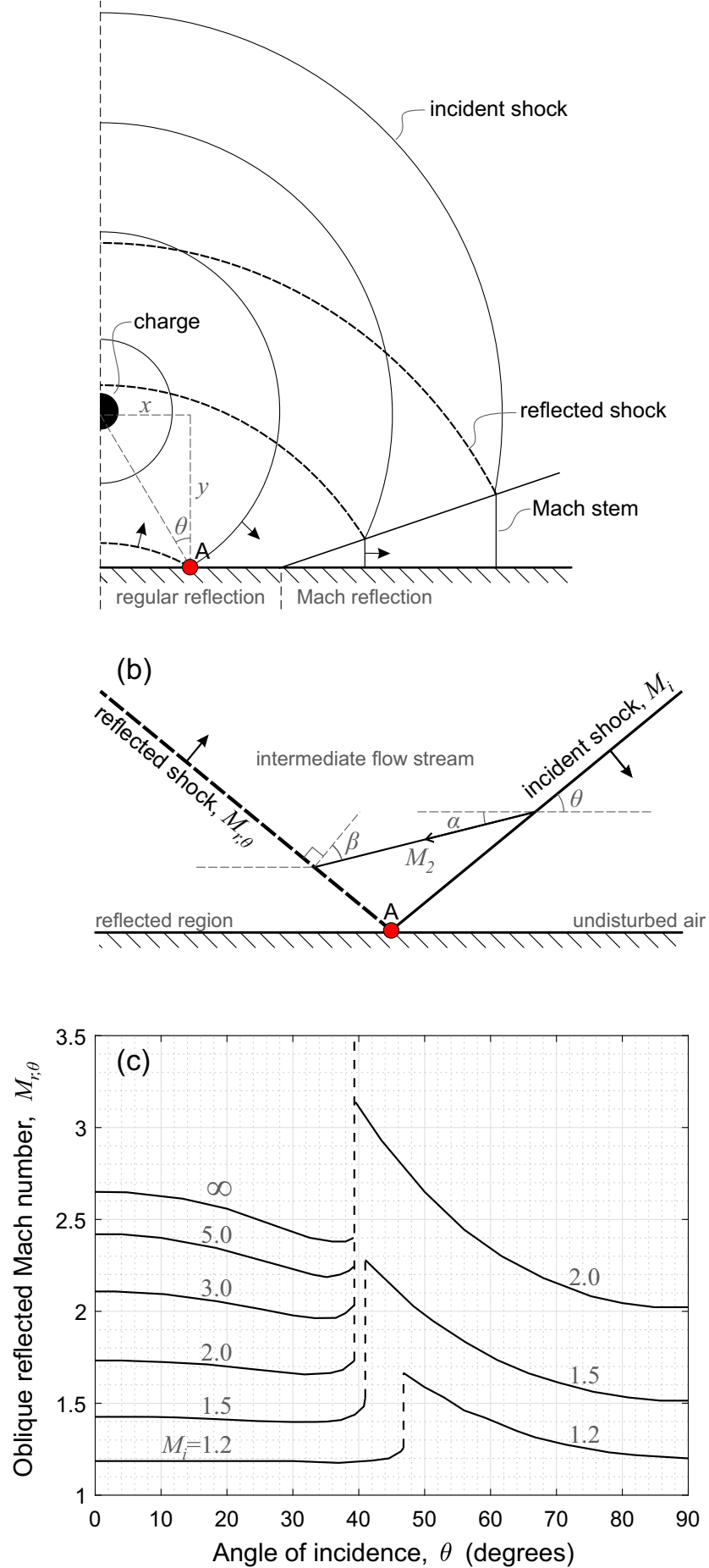

Fig. 9 a formation of the Mach Stem following impingement of an incident wave on a rigid reflecting surface, and definition of angle of incidence $\theta ; \mathbf{b}$ steady-flow counterpart of oblique shock reflection; c oblique reflected Mach number as a function of angle of incidence, with discontinuities showing transition from regular to Mach reflection. (a) adapted from [43], (b) and (c) adapted from [44] 


\section{Comparison to Directly Measured Peak Pressures}

Directly measured overpressure distributions from the HPB tests described in "Experimental Work" are used to assess the veracity of the HSV overpressure predictions, determined from the experimentally derived velocity-radius relationship and the [44] analytical framework outlined above in "Reflected Pressure Calculations from Fireball Velocity". Two configurations are compared: 100 g PE4 spheres detonated at $80 \mathrm{~mm}$ and $380 \mathrm{~mm}$ measured orthogonally from the charge centre to the centre of the rigid reflecting surface. It is worth reiterating that reflected pressure distributions are derived from incident Mach number measurements only, through interpolation of the derived velocity-radius relationship.

HPB measurements for each individual bar, and the mean value at each measurement location, are shown graphically alongside the HSV predictions in Fig. 10a and $b$ for the $80 \mathrm{~mm}$ and $380 \mathrm{~mm}$ tests respectively ${ }^{3}$, allowing for a more direct comparison between peak pressure distributions [27, 28]. HSV predictions were evaluated at $1 \mathrm{~mm}$ increments along the target surface. Whilst it is not possible to provide error bars of the HSV experimental measurement technique in isolation, pressure predictions are also provided by assuming incident velocities $\pm 1 \sigma$ and $\pm 2 \sigma$ from the mean at each gauge location, using an aggregate of the time-varying relative standard deviations of each test, as presented in Fig. 7. It is suggested that this envelope primarily encapsulates the intrinsic variability in near-field blast events, i.e. it is a measure of the typical experimental spread of results due to physical features of the fireball as it expands. This is perhaps best illustrated with reference to the right-hand column of Fig. 3, where features at the fireball/air interface are considerably larger than a single pixel and hence any calibration or tracking inaccuracies will be second order.

Generally the HSV pressure distributions closely follow those from the HPB measurements, with the magnitude and shape of the pressure distribution curve being well predicted. In the $80 \mathrm{~mm}$ stand-off tests, 50 of the $51 \mathrm{HPB}$ peak pressures lie within $\pm 2 \sigma$ of the HSV predictions, whereas in the $380 \mathrm{~mm}$ tests 41 out of 51 of the HPB peak pressures lie within $\pm 2 \sigma$ of the HSV predictions.

Localised increases in reflected pressure can be seen in the HSV predictions for the $80 \mathrm{~mm}$ tests at a distance from the plate centre, $x$, of approximately $65 \mathrm{~mm}(\theta=$ $40^{\circ}$ ) as a result of the Mach stem. The pressure increase

\footnotetext{
${ }^{3}$ The apparent decrease in pressure at the centre of the plate in the $380 \mathrm{~mm}$ tests is as a result of averaging over a limited dataset (only 1 Hopkinson pressure bar per test, giving a total of 3 data points) rather than being a genuine physical feature of the loading distribution
}

caused by the Mach Stem persists only for a short duration before being followed by a marked temporal decrease in pressure and rapid return to regular reflection conditions thereafter [43]. The disagreement between HPB and HSV pressures at $75 \mathrm{~mm}$ from the plate centre in Fig. 10a is likely due to a limitation of the HPB technique. Propagating pressure signals will exhibit a slight loss of definition of transient pressure features as a result of Pochhammer-Chree dispersion [45, 46], and hence peak pressures recorded using the HPB technique may be a slight underestimation of the true peak reflected pressure at that angle of incidence, i.e. in the region of Mach reflection. In a recent study, dispersion was shown to affect peak pressure recordings by up to 5\% for normal reflection [47], and it is suggested that this loss could be greater for irregular/Mach reflection on account of the transient, high-frequency components of the Mach Stem.

The HSV predictions appear to match the upper bound of the HPB pressure measurements for the $380 \mathrm{~mm}$ tests in Fig. 10b. It is likely that the mean velocity-radius relationship in Fig. 8 has been slightly skewed by the growth of instabilities and turbulent features as discussed in "Fireball Radius and Velocity Measurements". However, the results still compare well to the general range of peak pressures recorded at any distance from the plate centre, albeit the HSV technique is generally less able to match the bottom-end of the HPB data.

The results presented in Fig. 10 are compiled in Table 1 along with incident (measured) and reflected (calculated) Mach numbers. As previously, $y, x$, and $r$ are stand-off distance, distance from the plate centre, and slant distance to the measurement point, which is assumed to be exactly equal to the fireball radius at the moment of impingement after [21]. Maximum and mean HPB peak pressures are provided for comparative purposes. For the $80 \mathrm{~mm}$ standoff tests, the HSV data all lie within $10 \%$ of the mean HPB data at each measurement location, with the exception of the $x=75 \mathrm{~mm}$ data on account of the Mach Stem as discussed previously. Conversely, the HSV data lie within $10 \%$ of the maximum recorded HPB data at each measurement location and are typically $15-20 \%$ greater than the mean.

Peak incident pressures determined from equation (5) are also provided in Table 1. Taking the ratio of calculated reflected and incident pressures from analysis of the HSV data allows the reflection coefficient to be calculated. Here, the reflection coefficient ranges from 7.82-3.30 for the $80 \mathrm{~mm}$ stand-off case, and ranges from $6.41-6.08$ for the $380 \mathrm{~mm}$ case, which would be expected since it is a function of both angle of incidence and incident shock strength. Remembering that the velocity-radius relationship was derived for incident conditions, the values of reflection 

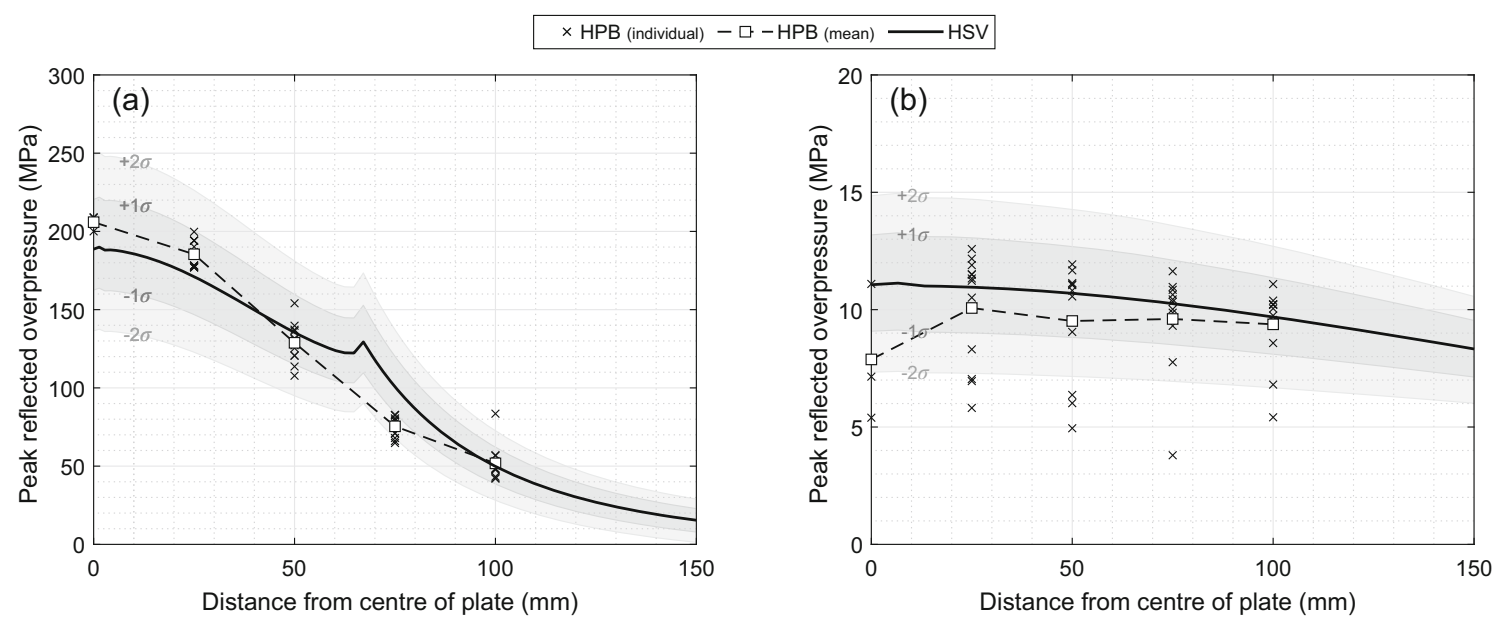

Fig. 10 Comparison of measured pressure distributions using Hopkinson pressure bar (HPB) and high speed video (HSV) predictions: a 80 mm stand-off distance; $\mathbf{b} 380 \mathrm{~mm}$ stand-off distance. Shaded regions indicate $\pm 1 \sigma$ and $\pm 2 \sigma$ bounds

coefficient provide further insight into the accuracy of the HSV method: this article has demonstrated that it is possible to use empirical measurement and RankineHugoniot theory to accurately predict distributed pressures up to a factor of $\sim 8$ greater than the incident pressure of the propagating blast wave. Whilst the pressure predictions may be improved by taking real gas effects into account, the assumption of ideal gas behaviour for the range of pressures in this article has been shown to be sufficiently accurate.

\section{Outlook}

The results presented in this article have potentially significant implications for the measurement of extremely high pressure near-field blast loads, in that it has been shown that there is no longer the requirement to locate an obstacle in the path of a blast wave in order to measure the pressure distribution that the obstacle will be subjected to.

The HSV analysis, previously conducted for $80 \mathrm{~mm}$ and $380 \mathrm{~mm}$, was extended to study the distribution of reflected pressure for stand-off distances, $y$, of $50-300 \mathrm{~mm}$ at increments of $5 \mathrm{~mm}$, for distances out to $250 \mathrm{~mm}$ from the plate centre, $x$, again at $5 \mathrm{~mm}$ increments, following detonation of a $100 \mathrm{~g}$ spherical PE4 explosive. The results are shown in Fig. 11. This analysis enables reflected pressure distributions to be estimated for a range of target sizes and stand-off distances (scaled target sizes of up to $0.54 \mathrm{~m} / \mathrm{kg}^{1 / 3}$ and scaled distances $0.11-0.65 \mathrm{~m} / \mathrm{kg}^{1 / 3}$ ) by reading reflected overpressure values off for a given value of stand-off. Hopkinson-Cranz scaling [25] can be used to

Table 1 Compiled peak oblique reflected pressures, $p_{r, \theta}$, from Hopkinson pressure bar (HPB) measurements and high speed video (HSV) predictions with associated input values

\begin{tabular}{|c|c|c|c|c|c|c|c|c|c|}
\hline \multirow[t]{3}{*}{$y(\mathrm{~mm})$} & \multirow[t]{3}{*}{$x(\mathrm{~mm})$} & \multirow[t]{3}{*}{$r(\mathrm{~mm})$} & \multirow[t]{3}{*}{$\theta\left(^{\circ}\right)$} & \multirow[t]{3}{*}{$M_{i}(-)$} & \multirow[t]{3}{*}{$p_{i}(\mathrm{MPa})$} & \multirow[t]{3}{*}{$M_{r}(-)$} & \multicolumn{3}{|c|}{$p_{r, \theta}(\mathrm{MPa})$} \\
\hline & & & & & & & \multirow[t]{2}{*}{ HSV } & \multicolumn{2}{|l|}{ HPB } \\
\hline & & & & & & & & Max & Mean \\
\hline 80 & 0 & 80 & 0 & 14.32 & 24.12 & 2.613 & 189.0 & 209.1 & 205.9 \\
\hline 80 & 25 & 83.82 & 17.35 & 14.06 & 23.25 & 2.535 & 171.4 & 199.8 & 185.4 \\
\hline 80 & 50 & 94.34 & 32.00 & 13.37 & 21.00 & 2.378 & 135.6 & 154.1 & 128.8 \\
\hline 80 & 75 & 109.7 & 43.15 & 12.41 & 18.10 & 2.231 & 101.2 & 82.75 & 75.44 \\
\hline 80 & 100 & 128.1 & 51.34 & 11.36 & 15.13 & 1.718 & 50.11 & 83.53 & 51.78 \\
\hline 380 & 0 & 380 & 0 & 4.110 & 1.878 & 2.314 & 12.04 & 11.10 & 7.880 \\
\hline 380 & 25 & 380.8 & 3.76 & 4.103 & 1.870 & 2.310 & 11.96 & 12.58 & 10.07 \\
\hline 380 & 50 & 383.3 & 7.50 & 4.078 & 1.846 & 2.298 & 11.60 & 11.93 & 9.517 \\
\hline 380 & 75 & 387.3 & 11.16 & 4.040 & 1.809 & 2.274 & 10.99 & 11.63 & 9.602 \\
\hline 380 & 100 & 392.9 & 14.74 & 3.989 & 1.759 & 2.254 & 10.74 & 11.09 & 9.375 \\
\hline
\end{tabular}



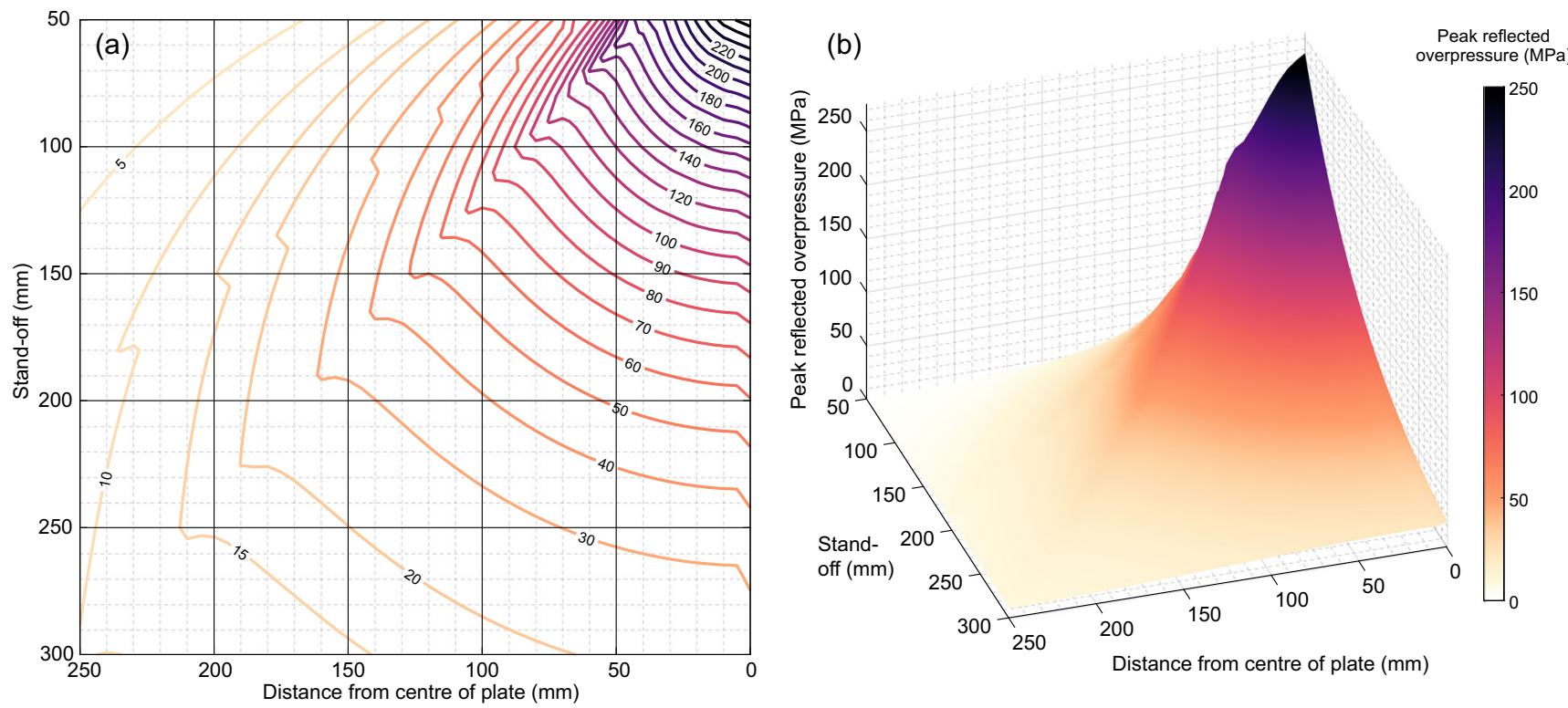

Fig. 11 Variation of peak reflected pressure (in MPa) with stand-off and distance from plate centre for a $100 \mathrm{~g}$ spherical PE4 explosive, through solution of equations (2) and (6-10): a contour plot; b surface

express the results at a different scale. It should be noted that the effects of blast wave clearing around the target edge have not been included in this analysis [48], however clearing is negligible in the near-field and can be neglected without a significant loss of accuracy [49].

Pressure distribution charts of this nature can be generated for any type and shape of explosive, provided the velocity-radius relationship is known, i.e. determined from high speed video analysis as in this article. This will allow the analyst to generate a suite of empirical loading distributions for any number of stand-off distances and target sizes from only a single test or a small number of repeat tests.

When evaluating structural response to blast loads it is often necessary to quantify the distribution of peak specific impulse (integral of the pressure history with respect to time) in addition to peak pressure distribution. Whilst quantification of specific impulse from HSV is an active area of research for the current authors and others [14], there are many applications where peak pressure is dominant. In particular, peak pressure distributions can provide validation data for numerical modelling, as well as being used to inform experimentalists of the typical pressures they should design their recording equipment to withstand. For example, a HPB with perimeter-mounted strain gauges should remain elastic (to facilitate repeat use, and to ensure the entire signal propagates as an elastic wave), and the gauges should operate within their calibrated range and remain bonded to the HPB. Clearly, these are all dictated by the magnitude of peak pressure, and therefore the methods presenting in this article could be used by researchers to provide a benchmark prior to additional experimental work.

\section{Summary and Conclusions}

This article presents the results from high speed video analysis of near-field explosive detonations. Edge detection algorithms were used to track the outer surface of the explosive fireball in order to determine a relationship between fireball surface velocity (i.e. attached shock wave velocity) and fireball radius. The measured velocity behaviour showed two clear stages of Rayleigh-Taylor [35, 36] and RichtmyerMeshkov [37, 38] surface instabilities: emergence and growth. The emergence stage persists up to a distance of approximately 10 charge radii, and is characterised by lowlevel and largely deterministic/repeatable variations in fireball surface velocities. At distances greater than 10 charge radii the growth stage is entered, which is characterised by a divergence in fireball surface velocities and higher variability in blast pressures.

Rankine-Hugoniot theory was used to convert the measured incident shock wave velocities into reflected pressure distributions. Whilst image tracking techniques have been used previously to measure variations in incident blast pressures with stand-off distance, this technique has not previously been used to evaluate near-field reflected pressures, nor how they are distributed along a target surface. 
Two test cases were considered: $100 \mathrm{~g}$ PE4 spheres detonated at stand-off distances (from the centre of the charge) of $80 \mathrm{~mm}$ and $380 \mathrm{~mm}$. The results compared well with directly measured pressure distributions using the apparatus described by [11], and were typically within $10 \%$ of the mean experimental value at each measurement location on the surface of a nominally rigid target.

The analysis method was extended to develop estimations of peak reflected pressure distribution for a range of near-field stand-off distances (scaled target sizes of up to $0.54 \mathrm{~m} / \mathrm{kg}^{1 / 3}$ and scaled distances $0.11-0.65 \mathrm{~m} / \mathrm{kg}^{1 / 3}$ ). Using this technique, it is possible to evaluate the blast loading on a structure using a single velocity-radius relationship. The results herein demonstrate that it is possible to accurately estimate reflected pressure distributions $\left(p_{r}>\right.$ $180 \mathrm{MPa}$ ) for situations where the blast wave possesses only free-air incident blast conditions ( $p_{i}<25 \mathrm{MPa}$ ). Thus, it is no longer necessary to locate sensitive recording equipment close to the source of the explosion in order to accurately measure the peak pressure acting on a surface.

Acknowledgements The authors wish to thank the technical staff at Blastech Ltd. for their assistance in conducting the experimental work. A. Tyas gratefully acknowledges funding from the Royal Academy of Engineering Research Chair Fellowship scheme.

Funding Information Experimental work reported in this paper was funded by Defence Science and Technology Laboratory and performed by Blastech Ltd. under contract no. BT-212.

\section{Compliance with Ethical Standards}

Conflict of interests The authors declare that they have no conflict of interest.

Open Access This article is licensed under a Creative Commons Attribution 4.0 International License, which permits use, sharing, adaptation, distribution and reproduction in any medium or format, as long as you give appropriate credit to the original author(s) and the source, provide a link to the Creative Commons licence, and indicate if changes were made. The images or other third party material in this article are included in the article's Creative Commons licence, unless indicated otherwise in a credit line to the material. If material is not included in the article's Creative Commons licence and your intended use is not permitted by statutory regulation or exceeds the permitted use, you will need to obtain permission directly from the copyright holder. To view a copy of this licence, visit http:// creativecommonshorg/licenses/by/4.0/.

\section{References}

1. Kingery CN, Bulmash G (1984) Airblast parameters from TNT spherical air burst and hemispherical surface burst, Technical Report ARBRL-TR-02555, U.S Army BRL Aberdeen Proving Ground, MD, USA

2. Kingery CN (1966) Airblast parameters versus distance for hemispherical TNT surface bursts, Technical Report ARBRL-TR1344, U.S Army BRL Aberdeen Proving Ground, MD, USA
3. Cheval K, Loiseau O, Vala V (2010) Laboratory scale tests for the assessment of solid explosive blast effects. Part i: Free-field test campaign. J Loss Prev Process Ind 23(5):613-621

4. Cheval K, Loiseau O, Vala V (2012) Laboratory scale tests for the assessment of solid explosive blast effects. Part II: Reflected blast series of tests. J Loss Prev Process Ind 25(3):436-442

5. Rigby SE, Tyas A, Fay SD, Clarke SD, Warren JA (2014a) Validation of semi-empirical blast pressure predictions for far field explosions - is there inherent variability in blast wave parameters? In: 6th International conference on protection of structures against hazards, Tianjin, China

6. Esparza E (1986) Blast measurements and equivalency for spherical charges at small scaled distances. Int J Impact Eng 4(1):23-40

7. Bogosian D, Ferritto J, Shi Y (2002) Measuring uncertainty and conservatism in simplified blast models. In: 30th explosives safety seminar, Atlanta, GA, USA

8. Rigby S, Tyas A, Clarke S, Fay S, Reay J, Warren J, Gant M, Elgy I (2015a) Observations From preliminary experiments on spatial and temporal pressure measurements from Near-Field free air explosions. Int J Protective Struct 6(2):175-190

9. Shin J, Whittaker AS, Cormie D (2015) Incident and normally reflected overpressure and impulse for detonations of spherical high explosives in free air. J Struct Eng 141(12):04015057

10. Leiste HU, Fourney WL, Duff T (2013) Experimental studies to investigate pressure loading on target plates. Blasting \& Fragmentation 7(2):99-126

11. Clarke SD, Fay SD, Warren JA, Tyas A, Rigby SE, Elgy I (2015) A Large scale experimental approach to the measurement of spatially and temporally localised loading from the detonation of shallow-buried explosives. Meas Sci Technol 26:015001

12. Dewey JM (1971) The properties of a blast wave obtained from an analysis of the particle trajectories. Proceedings of the Royal Society of London. Series A, Mathematical and Physical Sciences 324(1558):275-299

13. Dewey JM, Gaydon AG (1964) The air velocity in blast waves from T.N.T. explosions. Proceedings of the Royal Society of London. Series A, Mathematical and Physical Sciences 279(1378):366-385

14. Biss MM, McNesby KL (2014) Optically measured explosive impulse. Experiments in Fluids 55(6):1749-1-8

15. Hargather MJ, Settles GS (2007) Optical measurement and scaling of blasts from gram-range explosive charges. Shock Waves 17(4):215-223

16. Hargather MJ (2013) Background-oriented schlieren diagnostics for large-scale explosive testing. Shock Waves 23(5):529-536

17. Mizukaki T, Wakabayashi K, Matsumura $T$, Nakayama $K$ (2014) Background-oriented schlieren with natural background for quantitative visualization of open-air explosions. Shock Waves 24(1):69-78

18. Anderson JG, Parry BSL, Ritzel DV (2016) Time dependent blast wave properties from shock wave tracking with high speed video. In: 24th military aspects of blast and shock, Halifax, Nova Scotia, Canada

19. Courtiaud S, Lecysyn N, Damamme G, Poinsot T, Selle L (2019) Analysis of mixing in high-explosive fireballs using small-scale pressurised spheres. Shock Waves 29(2):339-353

20. Samuelraj IO, Jagadeesh G, Kontis K (2013) Micro-blast waves using detonation transmission tubing. Shock Waves 23(4):307316

21. Jenkins CM, Ripley RC, Wu C, Horie Y, Powers K, Wilson WH (2013) Explosively driven particle fields imaged using a high speed framing camera and particle image velocimetry. Int $\mathbf{J}$ Multiphase Flow 51:73-86 
22. Settles GS (2001) Schlieren and Shadowgraph techniques: Visualizing Phenomena in Transparent Media. Springer, Berlin

23. McNesby KL, Biss MM, Benjamin RA, Thompson RA (2014) Optical measurement of peak air shock pressures following explosions. Propellants, Explosives, Pyrotechnics 39(1):59-64

24. McNesby KL, Homan BE, Benjamin RA, Boyle VM, Densmore JM, Biss MM (2016) Quantitative imaging of explosions with high-speed cameras. Rev Sci Inst 87(5):051301-1-14

25. Baker WE (1973) Explosions in air. University of Texas Press, Austin

26. US Army Materiel Command (1974) Explosions in air part one. Department of the Army, Alexandria. AMCP-706-181

27. Rigby S, Fay S, Clarke S, Tyas A, Reay J, Warren J, Gant M, Elgy I (2016) Measuring spatial pressure distribution from explosives buried in dry leighton buzzard sand. Int J Impact Eng 96:89-104

28. Taylor LC, Fourney WL, Leiste HU (2010) 'Pressures on targets from buried explosions'. Int J Blasting \& Fragmentation 4(3):165192

29. Hopkinson B (1914) A method of measuring the pressure produced in the detonation of high explosives or by the impact of bullets. Philosophical Transactions of the Royal Society of London, Series A, Containing Papers of a Mathematical or Physical Character 213:437-456

30. Tyas A (2019) Blast loading from high explosive detonation: what we know and what we don't know. In: 13th International conference on shock and impact loads on structures, Guangzhou, China

31. Bogosian D, Yokota M, Rigby S (2016) TNT Equivalence of C-4 and PE4: a review of traditional sources and recent data. In 24th military aspects of blast and shock, Halifax, Nova Scotia, Canada

32. Rigby SE, Tyas A, Curry RJ, Langdon GS (2019) Experimental measurement of specific impulse distribution and transient deformation of plates subjected to near-field explosive blasts. Exp Mech 59(2):163-178

33. Canny J (1986) A Computational approach to edge detection. IEEE Trans Pattern Anal Mach Intell 8(6):679-698

34. Cui S, Wang Y, Qian X, Deng Z (2013) Image processing techniques in shockwave detection and modeling. J Signal Inform Process 4(3B): 109-113

35. Rayleigh Lord (1882) Investigation of the character of the equilibrium of an incompressible heavy fluid of variable density. Proc Lond Math Soc 4(1):170-177
36. Taylor GI (1065) The instability of liquid surfaces when accelerated in a direction perpendicular to their planes. I. Proceedings of the Royal Society a: Mathematical Phys Eng Sci 201:192-196

37. Meshkov EE (1969) Instability of the interface of two gases accelerated by a shock wave. Fluid Dynamics 4:101-104

38. Richtmyer RD (1960) Taylor instability in a shock acceleration of compressible fluids. Commun Pur Appl Math 13:297-319

39. Needham CE (2010) Blast Waves. Springer, Berlin

40. Schoutens JE (1979) Review of large HE charge early-time detonation anomalies Technical Report DASIAC SR 182. Defense Nuclear Agency, Washington

41. Tyas A, Reay JJ, Fay SD, Clarke SD, Rigby SE, Warren JA, Pope DJ (2016) 'Experimental studies of the effect of rapid afterburn on shock development of near-field explosions'. Int J Protective Struct 7(3):456-465

42. Pontalier Q, Loiseau J, Goroshin S, Frost DL (2018) Experimental investigation of blast mitigation and particle-blast interaction during the explosive dispersal of particles and liquids. Shock Waves 28(3):489-511

43. Rigby SE, Fay SD, Tyas A, Warren JA, Clarke SD (2015b) Angle of incidence effects on far-field positive and negative phase blast parameters. Int J Protective Struct 6(1):23-42

44. Kinney GF, Graham KJ (1985) Explosive Shocks in Air. Springer, USA

45. Rigby S, Barr A, Clayton M (2018) A review of PochhammerChree dispersion in the Hopkinson bar. Proceedings of the Institution of Civil Engineers: Engineering and Computational Mechanics 171(1):3-13

46. Tyas A, Watson AJ (2001) An investigation of frequency domain dispersion correction of pressure bar signals. Int J Impact Eng 25(1):87-101

47. Barr A, Rigby S, Clayton M (2020) Correction of higher mode Pochhammer-Chree dispersion in experimental blast loading measurements. Int J Impact Eng 139(103526):1-8

48. Rigby SE, Tyas A, Bennett T, Fay SD, Clarke SD, Warren JA (2014b) A Numerical investigation of blast loading and clearing on small targets. Int J Protective Struct 5(3):253-274

49. Shin J, Whittaker AS (2019) Blast-wave clearing for detonations of high explosives. J Struct Eng 145(7):04019049

Publisher's Note Springer Nature remains neutral with regard to jurisdictional claims in published maps and institutional affiliations. 